\title{
Clinical Tomographic Correlations of 220 Patients with Neurocisticercosis, Bahia, Brazil
}

\author{
Antônio de Souza Andrade-Filho' ${ }^{1}$, Luiz Frederico da Silva Figuerôa ${ }^{2}$ and Victor Mascarenhas Andrade-Souza ${ }^{3}$ \\ ${ }^{1}$ Department of Neuropsychiatry - FAMEB (UFBA); ${ }^{2}$ Foundation of Neurology and Neurosurgery - Brain Institute; ${ }^{3}$ School of Medicine and \\ Public Health - EMSP; Salvador, BA, Brazil
}

\begin{abstract}
Neurocysticercosis (NCC) is a common parasitic disease in our region, presenting diversity of neurological symptoms and signs. The present study has as primary objective an evaluation of the NCC's clinical and epidemiological profile within Bahia State, by means of a prospective study of 220 patients diagnosed from March 1988 to March 1999, with a follow-up of six months. Exams, such as Computed Cranial Tomography Scan (CT), Cerebral Spine Fluid (CSF) and Electroencephalogram (EEG), were accomplished in three distinct moments of these patients' evolution: at starting or diagnostic point (zero time), at after-intervention period (one month after treatment), and at control period (six months after treatment).
\end{abstract}

Key-Words: Neurocysticercosis, computadorized tomography, epidemiology, clinical.

Cysticercosis is a parasitosis, typical of developing regions, which is associated to poor hygiene and basic sanitation conditions. From the epidemiological point of view, Neurocysticercosis can be found disseminated in different parts of the world, and becomes extremely important in the social and economic profile of the affected areas, because $75 \%$ of the patients are found at their fully productive age [1].

In Brazil, the greatest prevalence can be found in the states of Paraná, São Paulo, Minas Gerais, Rio de Janeiro and Bahia [2]. It is most common among adults who are on the third and fourth decade of age. There is no preference, though, for a definite sex or social class. Its most common clinical manifestations are epileptic and hypertensive syndromes, being the cause for late epilepsy in different endemic areas. Mental alterations are frequent in the disease's course, schizophrenic syndromes, humor disturbances and cognitive deterioration have been described. The present study analyzes patients bearing Neurocysticercosis (NCC), by associating its clinical presentation with lesions found at the Cranium Computerized Tomography (CT).The CT results were divided into 4 stages : stage I - initial cyst, with no inflammatory response stimulation or with a minimal inflammatory reaction; stage II - edema surrounded cyst and intense inflammatory reaction, forming a granuloma; stage III - granuloma under degenerative process; and stage IV - calcified granuloma. The treatment was based on the disease's activity criteria, and consisted of prescribing Anticysticercotic drugs (Praziquantel or Albendazole), Corticoid and Symptomatic drugs.

\section{Materials and Methods}

The study has utilized a protocol, consisting of a questionnaire containing data referring to social and economical, demographic and neurological variables,

Received on 20 September 2006; revised 16 January 2007.

Address for correspondence: Dr. Antonio Andrade. Foundation of Neurology and Neurosurgery - Brain Institute. Rua Deocleciano Barreto, 10. Zip code: 40140-110 - Salvador, BA. E-mail: andradeneuro@uol.com.br.

The Brazilian Journal of Infectious Diseases 2007;11(1):114-117. (C) 2007 by The Brazilian Journal of Infectious Diseases and Contexto Publishing. All rights reserved. concerning the clinical forms of presentation, complementary exams (Complete CSF study and Axial Computerized Tomography and/or Magnetic Resonance) as well as Therapeutics. This protocol has been applied in the services of the public and private network since March 1988. The criteria for including patient in the protocol is the NCC diagnosis, which must be confirmed by the CSF exam (IFI, HAI and ELISA), as well as by imaging (CT).

The data referring to the 220 patients have been evaluated, having been collected from March 1988 to March 1999. Those obtained data have been analyzed by the SPSS statistical program (version 6.0 for Windows).

\section{Results}

The results have demonstrated that, regarding demographic data, 125 patients (56.8\%) were males, while 95 (43.2\%) were females, having an equivalent distribution among the white, mulatto and black races (Figure 1). It has been observed that NCC has affected individuals with a mean age of 30.3 years, varying from 2 to 84 years of age. About one half of the patients ( $49.1 \%$ or 108 patients) proved having had concluded the junior high school education, and were still studying, predominantly (21.9\% or 48 patients), followed by domestic occupation (14.6\% or 32 patients) and merchants (9.1\% or 20 patients).

As for the clinical manifestations, the convulsive crisis was the most important form, appearing in an isolate way, in $80.9 \%$ of the cases, while the psychiatric form appeared in $1.4 \%$, and the intracranial hypertension syndrome (ICHS) was present in $3.2 \%$ of the cases (Figure 2).

In general, the intracranial hypertension syndrome was present in $14.1 \%$ of the cases, while the psychiatric form, in $5.5 \%$ of them. The studied sample has not evidenced the presence of the medullar form.

As for the neurological variables, 180 patients (81.8\%) had displayed normal neurological examination. The sign found was hemiparesis isolated in 20 patients (9.1\%). Pure hemiplegia was present in 5 patients (2.3\%). The association between dysarthria and hemiparesis occurred in 2 patients ( $0.9 \%)$, while alterations of cranial pairs occurred in $6(2.8 \%)$ 
cases. The less frequent presentations are dysarthria, hemiparesis and/or cranial nerves, associated with cerebellar syndrome, with one patient for each case (Figure 3).

The correlation between clinical forms and tomography stages, in the initial time period, evidences a statistical significance between ICHS and stage I ( $p=0.02)$, as well as between the clinical and the convulsive forms and stage II ( $\mathrm{p}$ $=0.005$ ). There was no significant correlation between stages III and IV and the clinical forms.

By following the same principles, when the neurological variables are compared with the initial CT stages, it has been evidenced that the patients having a normal neurological exam have presented a greater association with stage IV $(p=0.001)$, while those patients bearing hemiparesis alone had a greater correlation with stage IV $(p=0.002)$. The remaining crossings, though, have not been significant ones.

As for the CSF findings at zero time, from one to six months, one can perceive a significant decrease in the number of inflammatory CSF, and a proportional increase of noninflammatory CSF, after a clinical intervention. There has been an evolution in the non-inflammatory CSF, going from 68.07\% to $87.50 \%$ and $93.60 \%$, as well as of inflammatory CSF going from $31.90 \%$ to $12.50 \%$ and $6.38 \%$ for the zero time, one month's period and six months' period, respectively. There has been improvement in the electroencephalic exams, carried out within the same periods of time. The EEG at zero time was normal in only $31 \%$ of the patients, and has evolved to a normal condition in $80 \%$ of the patients who have been reevaluated within a six months' period, displaying in proportional decrease of the abnormal EEG.

Figure 4, which has proved the CT results, at zero time, one month and six months, has evidenced that the zero time predominance stages are stages IV (44.03\% 0 and II (39.90\%). The value observed for stage I at zero time (24.70\%) has decreased gradually in one month (2.5\%) and six months (3.2\%), after follow-up start. The parasite's final evolution stage (stage IV) has increased from $44.03 \%$, at zero time, to $87.1 \%$, in the six months' period, denoting acceleration in the calcification process.

The most used therapeutic combination has been Albendazole, combined to corticoid and symptomatic drugs, in $59.5 \%$, followed by the isolated use of symptomatics, in $29.5 \%$. The praziquantel-corticoid and symptomatic association has benefited $4.5 \%$ of the cases, while one patient, who has not shown any improvement with Albendazole, has been, later on, treated with Praziquantel, Corticoids and Symptomatics, showing clinical improvement.

\section{Discussion}

According to the studied sample's demographic data, one can verify the disease's manifestation in both genders, with a 3:2 male/female rate and, with a greater frequency with the age ranging between the $2^{\text {nd }}$ and $3^{\text {rd }}$ decades. It reaches young adults and it is less common among children, these data agreeing with other reports found in the literature. This affection negative impact becomes evident for the most assailed subgroups, either from the occupational or from the educational point of view. It affects mostly the subgroup of students who are found at their active learning stage and who are impaired by this neurological disease and, due to convulsive seizures, it also endangers people who working on risk occupations, such as electricians, drivers, nursing aids, among others.

The present study, a a breakthrough in Bahia State, evidences that NCC is endemic in our environment.

Among NCC's clinical manifestations, the findings are compatible with other studies, confirming the convulsive crises, as the main symptom, presented either as isolate ones, or associated to other clinical forms. ICHS is the second most common symptom. The psychical manifestation has a 5.5\% prevalence, determining how important it is to include NCC in the differential diagnosis of organic causes of psychiatric disturbances. There was found a correlation between the psychiatric disturbances and presence of epilepsy $(\mathrm{p}=0.00)$, which cannot serve as a parameter, though, for determining whether epilepsy is a risk factor for psychiatric pathology or not. Studies have shown, however, that patients bearing convulsive crises, regardless of the underlying pathology, have the worst prognostic. This is probably due to the early installation of psychosis, or even due to the anti-convulsive drugs, which may represent a damaging effect to the patient's mental state. ICHS is a syndrome, most frequently determining the psychic manifestations at NCC. It is, therefore, a frequent psychic and neuropsychological affection among ambulatory patients, bearing NCC, and literature has demonstrated that those patients tend to present more affective disturbances that those displaying psychotic pictures. The main psychopathological trend, in this case, is that the symptoms are unspecific. One can observe that the presence of a neurological disease and the ICHS occurrence are risk factors for increasing psychiatric morbidity in those patients, for which additional studies are needed.

The patients' neurological exam was normal for most cases, probably because the cysticerco were located in the CNS's silent areas, or due to the low capacity of neuronal irritation. Out of the $18.6 \%$ patients who had had some findings in the neurological exam, most part of them presented hemiparesis, while they presented isolated hemiparesis, in the second place.

It can be noticed, therefore, that the typical NCC patient is the one having convulsive crises and a normal neurological exam, in spite of that disease being a pleomorphic one and being able to manifest itself under different forms.

The clinical-tomographic correlation has allowed evidencing that stage II is associated to the convulsive form, while the ICHS has only been associated to stage I. We can, thus, notice a greater trend towards the appearing of clinical forms at the disease's rather initial stages. We believe that the mere initial presence of cysticercosis in certain CNS areas may have caused ICHS (stage I), while the stimulation of the immune system, caused by the parasite, with a local 
Figure 1. Neurocysticercosis - Distribution per Race $(\mathrm{n}=220)$.

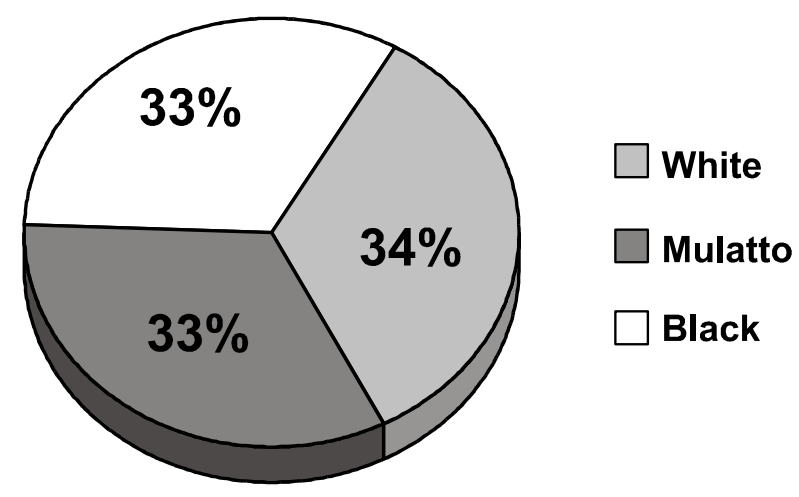

Figure 2. Neurocysticercosis - Clinical Forms (n=220).

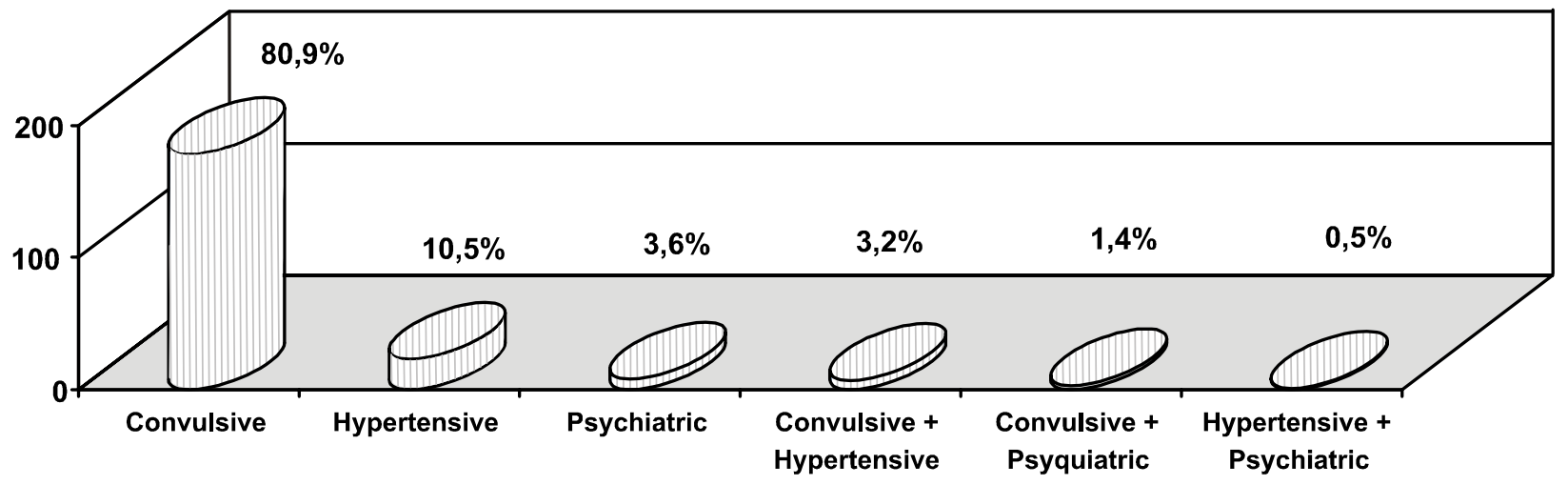

Figure 3. Neurocysticercosis - Neurological Variables in 220 Patients.

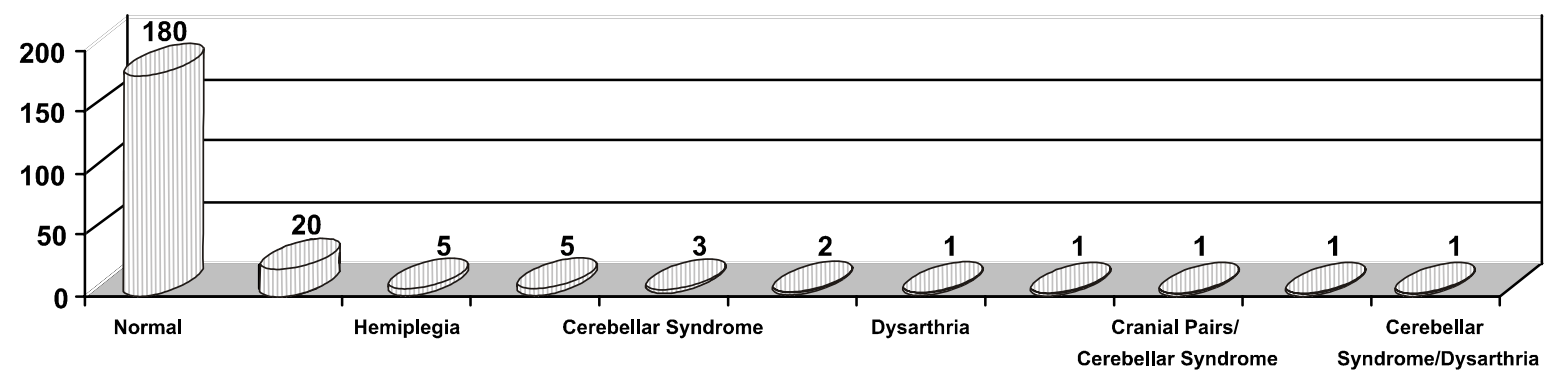

Figure 4. Neurocysticecosis - Computerized Tomography ( $n=220)$.

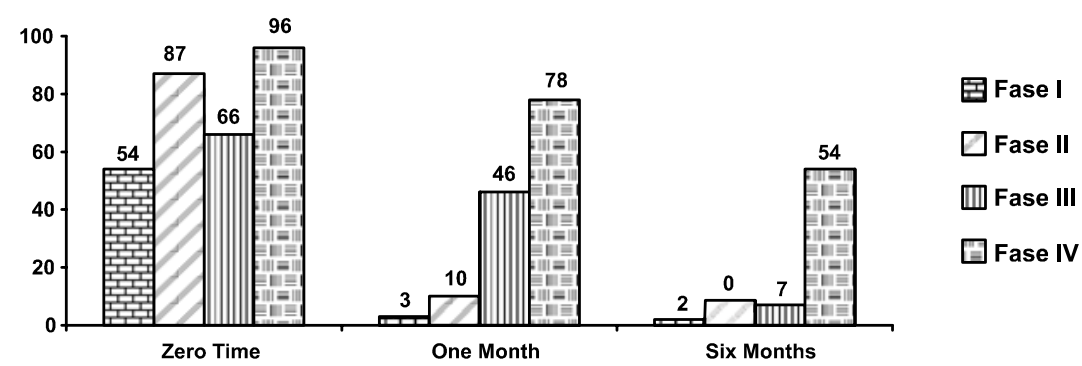

www.bjid.com.br 
inflammatory response, releasing of substances and important perilesional edema, might be responsible for the clinical manifestations found at stage II. As the inflammatory process is being solved, there can be noticed recurrence to some clinical manifestations, either because the parasite's growth has not been able to cause ICHS, or because the inflammatory disease had disappeared and the chemical mediators of the CNS have diminished, which increases the presence of convulsions. As for the pathogenesis of the convulsive crisis, the findings agree with reports by Del Bruto et al. [3-6], in which the active lesion has been emphasized as the main epileptogenic factor for this affection, since an important association between the convulsive form and stage II has been made. The presence of the perilesional gliosis (stage IV) probably acts as a secondary factor, and does not evidence any statistically significant correlation with the convulsive form, in our sample. In summary, ICHS has been related to stage I - in which the mere parasite's presence, either for its size or for being located in a noble area of the CNS, has a great importance in the ICHS physiopathology. As the process is being solved, it becomes quite possible that the diminishing of the affected area play an important part in the ICHS arousing. In the same way, there can be observed that stage II is an important point for the appearance of clinical manifestations, since it is the affection's most exuberant stage, when patients present convulsions, Psychic manifestations or ICHS.

It is now convenient stressing that, as it is a pleomorphic disease, it may display varied forms, and the findings do not have to be standardized.

Regarding the complementary exams, it is verified that at all proposed time periods, most of the CSF is non-inflammatory, and there is a trend to diminish the CSF inflammatory percentage with the evolution; while the EEG analysis evidences that at initial time, the abnormal EEG contributes greatly to the involved cases evolving to an improvement at the follow-up period. The CT evaluation at the initial time has proved to be important for all stages, while within a month's period, stages I and II have decreased significantly, and stage III presented a discrete growth, and stage IV has grown in proportion, according to its own evolution. In a six months' period (control time), it has been observed that the significant majority has evolved to stage IV, few patients remained at stage III, no patient was left at stage II, and one patient (who did not join the treatment) remained at stage I. It is quite possible that, if the follow-up duration is extended, all the patients found at stage IV could be evidenced. As it was described in literature, NC tends to take from 23 months to 7 years to calcify [7], it was demonstrated that in our sample the process was an accelerated one. In spite of the fact that treatment with anticyrcotic drugs does not extinguish the parasite, completely, from the CNS, in many cases, it can accelerate the calcification process [8-20]. As the CT findings at stage IV were statistically significant in relation to the normal neurological exam, we believe that the patients have been benefited by the treatment.

\section{References}

1. Flisser A. Neurocysticercosis in México. Parasitology Today 1988;4(5):131-6.

2. Andrade Filho A.S. Neurocisticercose - Aspectos Clínicos, Epidemiológicos e Diagnósticos - Estudo Prospectivo de 157 Pacientes na Região Nordeste-Bahia. Rio de Janeiro; 1997 (Tese de Doutorado).

3. Del Brutto O.H., Santibañez R., Noboa C.A., et al. Epilepsy due to neurocysticercosis; analisis of 203 patients. Neurology 1992;42:347-89.

4. Del Brutto O.H., Sotelo J. Neurocysticercosis: an up-date. R Inf Dis 1988;10(6):1075-87.

5. Del Brutto O.H., Sotelo J. Albendazole therapy for subarachnoid and ventricular cysticercosis; case report. J Neurosurg 1990;72:816-7.

6. Del Brutto O.H., Sotelo J., Aguirre R., et al. Albendazole therapy for giant subarachnoid cysticerci. Arch Neurol 1992;49:535-8.

7. Guerreiro M.M., Facure N.O., Guerreiro C.A.M. Aspectos da tomografia computadorizada craniana na neurocisticercose na infância. Arq Neuropsiquiatr São Paulo 1989;47(2):153-8.

8. Forlenza O.V., Vieira A.G.H. Filho, Nóbrega J.P.S., et al. Psychiatric manifestations of neurocisticercosis: a study of 38 patients from a neurology clinic in Brazil. J Neurol Neurosurg Psychiatry 1997;62:612-6.

9. Feinstein A., Ron M.A. Psychosis associated with demonstrable brain disease. Psychol Med 1990;20:793-803.

10. Tavares A.R. Jr. Psychiatric disorders in neurocisticerosis (Letter). Br J Psychiatric 1993;163:839.

11. Hoffman P., Saint-Paul M.C., Michiels J.F., et al. Atteinte isolée du système nerveux central au cours d'une cysticercose. Ann Pathol 1990;10(2):122-5.

12. Michel P., Michault A., Gruel J.C., Coulanges P. Le serodiagnostic de la cysticercose par ELISA et West blot; son intérêt et ses limites à Madagascar. Arch Inst Pasteur Madagascar 1990;57(1):115-42.

13. Webbe G. Huma cysticercosis: parasitology, pathology, clinical manifestations and available treatment. Pharmac Ther 1994;64:175-200.

14. Teitelbaum G.P., Otto R.J., Lin M., et al. Imaging of neurocysticercosis. AJR 1989;153:857-66.

15. Vazquez V., Sotelo J. The course of seizures after treatment for cerebral cysticercosis. N Eng J Med 1992;327(10):696-701.

16. Schantz P.M., Sarti-Gutierrez E. Diagnostic methods and epidemiologic surveillance of Taenia solium infection. Acta Leidensia 1987;57(2):153-63.

17. Robles C. Achados anatomopatológicos e causas de mortalidade tardia em enfermos de neurocisticercose tratados com praziquantel. JBM 1983;45(supl.4):63-71.

18. Minguetti G., Ferreira M.V.C. Ação de corticóides na fase aguda da neurocisticercose; nota preliminar. Arq Neuropsiquiatr São Paulo 1982;40(1):77-85.

19. Garcia H.H., Gilman R.H. Medical treatment of cysticercosis: Ineffective x Effective. Arch Neurol 1995;(52).

20. Arseni C., Cristescu A. Epilepsy due to cerebral cysticercosis. Epilepsia Amsterdam 1972;13:253-8. 